\title{
Incidence of fracture events with teriparatide: role of study design
}

\author{
I. Hernandez ${ }^{1}$ A. Piñera ${ }^{2}$
}

Received: 5 March 2015 / Accepted: 10 June 2015 / Published online: 20 June 2015

(C) International Osteoporosis Foundation and National Osteoporosis Foundation 2015

Dear Editor,

We read with interest the recent study by Bonafede et al. on the risk of fragility fractures among osteoporotic patients using teriparatide [1]. Based on US claims data, this study found that around $13 \%$ of teriparatide users experienced a fragility fracture in the two years following the initiation of teriparatide. In a further examination of the factors that affect the risk of fracture, Bonafede et al. found that the odds of experiencing a fracture were $31 \%$ higher ( $95 \% \mathrm{CI}, 17$ to $47 \%$ ) for non-persistent patients - those who had at least a 90-day gap in their teriparatide use during the 2-year follow-up period-than for persistent patients. Nevertheless, in order to better understand the role of persistence with teriparatide in the prevention of fragility fractures, several aspects of the study design need to be considered.

First, because study participants were not censored when they had a fracture, the temporal order of teriparatide discontinuation and fracture events cannot be determined. This is a major limitation of the study because patients may discontinue teriparatide after experiencing a fracture. In such a scenario, the observed association between non-persistence with teriparatide and risk of fracture would be a spurious one. Second, to select the sample, the authors required that study participants either had a diagnosis for osteoporosis in the year before teriparatide initiation or filled a prescription for an antiresorptive agent.

I. Hernandez

inh3@pitt.edu

1 Department of Health Policy and Management, Graduate School of Public Health, University of Pittsburgh, 130 De Soto St, Pittsburgh, PA 15261, USA

2 Department of Obstetrics and Gynecology, La Paz University Hospital, 261 Paseo de la Castellana, 28046 Madrid, Spain
Individuals with a diagnosis for several conditions were excluded from the analysis; however, osteopenia was not among the list of exclusion conditions. Consequently, it remains unclear whether the 1037 patients who had a diagnosis of osteopenia transitioned to osteoporosis in the year before treatment initiation. This is of special concern because the proportion of patients who had a diagnosis of osteopenia in the year before teriparatide initiation was unbalanced between persistent and non-persistent teriparatide users. Similarly, the authors acknowledged the unbalance of other baseline characteristics between two groups; however, they did not report those numbers. Because like age, some of the unbalanced factors may be associated with the risk of fracture, the results for the comparative risk of fracture between persistent and non-persistent patients may well be affected by selection biases. In order to clarify the potential for selection bias, it would be informative if the authors reported a comparison of baseline characteristics between persistent and non-persistent patients.

Nevertheless, we commend the authors for studying this important research question, and we hope they continue to investigate it while paying careful attention to the aspects outlined. Specifically, it would be informative to continue the investigation incorporating three changes in the study design: first, censoring individuals from the study sample when they experience a fracture; second, including in the study sample only patients with a diagnosis of osteoporosis in the year before teriparatide initiation; and third, using propensity score methods to balance patient characteristics between persistent and non-persistent patients.

\section{Reference}

1. Bonafede MM, Shi N, Bower AG, Barron RL, Grauer A, Chandler DB (2015) Teriparatide treatment patterns in osteoporosis and subsequent fracture events: a US claims analysis. Osteoporos Int 26(3): 1203-1212. doi:10.1007/s00198-014-2971-3 